\title{
A Comparison Study of Spinal Anesthesia with General Anesthesia in Cesarean Section
}

\author{
Rajeev Kumar Das ${ }^{1}$, Manu Seth ${ }^{2}$ \\ ${ }^{1}$ Assistant Professor, Department of Anaesthesia, TS Misra Medical College and Hospital Lucknow, Uttar Pradesh, India.
}

\section{Abstract}

Background: The present study was conducted to compare spinal anesthesia with general anesthesia in cesarean section. Subjects and Methods: The present study was conducted in the department of Anesthesiology. It comprised of 78 pregnant women with American Society of Anaesthesiologists (ASA) physical status grade I or II who were scheduled to undergo elective CS under SA. Patients were divided into 2 groups. In both groups, side-effects after surgery such as nausea, vomiting, headache, pain and other clinical symptoms were noted. Results: The mean WBC count in group I was 12.74 109/L and 10.62 109/L in group II which was significant $(\mathrm{P}<0.05)$. Other parameters such as mean $\mathrm{RBCs}$, mean hemoglobin and mean platelet count was non- significant ( $\mathrm{P}>0.05)$. In group I most common complication was fever (6) and hypotension (6) while in group II, fever (8) and hypotension (7). However, the difference was non- significant (P> 0.05). Conclusion: Both spinal anesthesia and general anesthesia showed variations in hematological parameters as well as complications.

Keywords: Cesarean, general anesthesia, spinal anesthesia.

Corresponding Author: Dr. Manu Seth Assistant Professor, Department of Anaesthesia, TS Misra Medical College and Hospital Lucknow, Uttar Pradesh, India.

Received: June 2019

Accepted: June 2019

\section{Introduction}

Caesarean section (CS) is now one of the most commonly performed major operations in women throughout the world. While regional or general anaesthesia (GA) are both acceptable for caesarean delivery, use of GA has decreased dramatically in the past few decades due to a higher risk of anaesthesia-related maternal mortality. ${ }^{[1]}$ As a consequence, spinal anaesthesia (SA) is now the technique of choice for CS. Although SA is generally well tolerated, it is still associated with considerable side effects, the most common of which is maternal hypotension, potentially endangering both mother and child. ${ }^{[2]}$

For balancing the pros and cons of the caesarean surgeries in relation to mother and her foetus, spinal anaesthesia should be preferred. Because of some selective advantages provided by SA over epidural anaesthesia, SA is preferred nowadays for performing elective caesarean sections. ${ }^{[3]}$ Evidence for maternal death in CS, especially due to excessive bleeding is rare and general anesthesia is not often considered in this regard. This is because of muscle relaxation and much less labor induction against spinal method. Furthermore, inhaled halogen contents in general anesthesia may induce more bleeding via suppression in uterine wall contraction and mother's consciousness. ${ }^{[4]}$ The present study was conducted to compare spinal anesthesia with general anesthesia in cesarean section.

\section{Subjects and Methods}

The present study was conducted in the department of Anesthesiology. It comprised of 78 pregnant women with American Society of Anaesthesiologists' (ASA) physical status grade I or II who were scheduled to undergo elective $\mathrm{CS}$ under SA. The study protocol was approved prior to the commencement of study. All patients were informed regarding the study and written consent was obtained.

General information such as name, age etc. was recorded in performa. Patients were divided into 2 groups. Group I received general anesthesia and group II received spinal anesthesia. Pre- operative assessment of the haematological parameters was done in both groups.

Caesarean section was performed in both the groups. Postsurgical symptoms were recorded. In both groups, sideeffects after surgery such as nausea, vomiting, headache, pain and other clinical symptoms were noted. Results were subjected to statistical analysis. $\mathrm{P}$ value less than 0.05 was considered significant.

\section{Results}

\section{Table 1: Distribution of patients}

\begin{tabular}{|l|l|l|}
\hline Total- 78 & Group I (GA) & Group II (SA) \\
\hline Groups & 39 & 39 \\
\hline Number
\end{tabular}


0

[Table 1] shows that group I received general anesthesia and group II received spinal anesthesia. Both groups had 39 patients each.

Table 2: Assessment of Post-operative hematological
parameters in both groups
\begin{tabular}{|l|l|l|l|}
\hline Parameters & Group I & Group II & P value \\
\hline Mean WBCs (x 109/L) & 12.74 & 10.62 & 0.05 \\
\hline Mean RBCs (x 109/L) & 5.6 & 5.2 & 0.12 \\
\hline Mean Haemoglobin (g/dL) & 9.6 & 9.8 & 0.74 \\
\hline Mean Platelet count (x 109/L) & 2.8 & 2.5 & 0.98 \\
\hline
\end{tabular}

[Table 2 \& Figure 1] shows that mean WBC count in group I was $12.74109 / \mathrm{L}$ and $10.62109 / \mathrm{L}$ in group II which was significant $(\mathrm{P}<0.05)$. Other parameters such as mean RBCs, mean hemoglobin and mean platelet count was nonsignificant $(\mathrm{P}>0.05)$.

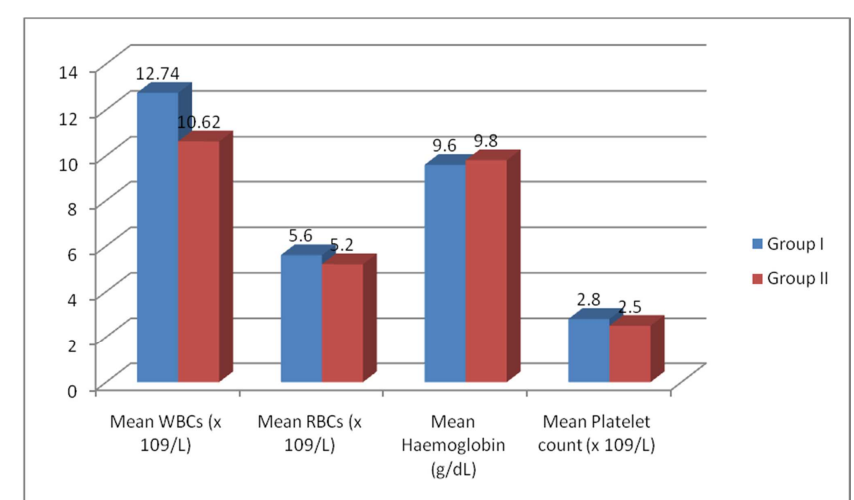

Figure 1: Post-operative hematological parameters in both groups

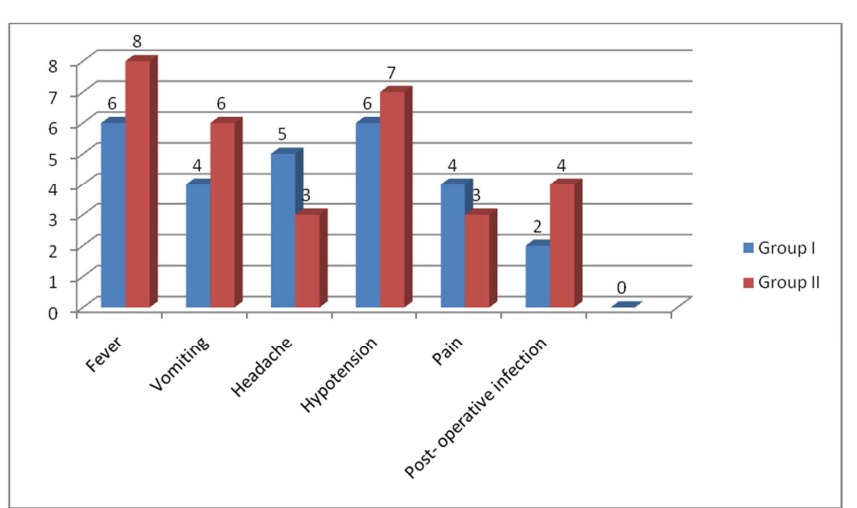

Figure 2: Assessment of complications in both groups

Graph II shows that in group I most common complication was fever (6) and hypotension (6) while in group II, fever (8) and hypotension (7). However, the difference was nonsignificant $(\mathrm{P}>0.05)$.

\section{Discussion}

Although both general and spinal anesthesia are used in elective cases of CS, the latter is much preferred, particularly when they need to keep mother awakes. Besides, mother aspiration and fetal distress would effectively reduce by spinal technique. ${ }^{[5]}$ Previous studies show a dilemma about labor bleeding and its causes when compare general and spinal anesthesia although the majority of authors determine more bleeding in general technique. However; still a lot of controversy exists regarding the negative or positive effects of spinal anaesthesia on the post-surgical analgesic effects in mothers undergoing caesarean sections. ${ }^{[6,7]}$ The present study was conducted to compare spinal anesthesia with general anesthesia in cesarean section.

In present study, group I received general anesthesia and group II received spinal anesthesia. Both groups had 39 patients each. Ajay8 in his study found that before surgery, mean WBCs (x 109/L) concentrations in patients undergoing GA and SA was 9.85 and 9.92 respectively. Mean RBCs (x 109/L) concentrations were 4.12 and 4.18 in patients undergoing GA and SA respectively before surgery. Nonsignificant results were obtained while comparing the mean WBC concentration, mean RBC concentration, mean hemoglobin and other hematological parameters in between the two study groups before the surgery. After surgery, mean WBCs (x 109/L) concentrations in patients undergoing GA and SA was 12.21 and 10.41 respectively. Statistically significant differences were obtained while comparing the mean WBC concentration in the two study groups when measured after the surgery. Pain and hypotension was the most common side effect prevalent in patients undergoing caesarean sections under both GA and SA.

We found that mean WBC count in group I was 12.74 109/L and $10.62109 / \mathrm{L}$ in group II which was significant $(\mathrm{P}<0.05)$. Other parameters such as mean RBCs, mean hemoglobin and mean platelet count was non- significant. In group I most common complication was fever (6) and hypotension (6) while in group II, fever (8) and hypotension (7).

Veneziani et al, ${ }^{[9]}$ found that all the elective CS with 38-40 weeks gestational age enrolled via easy sampling before being divided into two groups of general and spinal anesthesia. Patients' hemoglobin and HCT in addition to blood pressure were the major factors which were checked and compared between the groups. HB fell significantly more in patients with general anesthesia, especially at the range of 1-2 g/dl after 6 and 24 hours of CS. Around $91 \%$ of GA and more than $50 \%$ of SA had middle changes in HB and HCT. These changes were significantly different between GA and SA. The two groups were simply similar according to greater changes including $2-3 \mathrm{~g} / \mathrm{dl}$ in HB or 6-9 in HCT and contain a minor part of the patients. The present study indicated that bleeding and decreased HB and HCT occur significantly less in spinal anesthesia comparing to general anesthesia.

\section{Conclusion}

Both spinal anesthesia and general anesthesia showed variations in hematological parameters as well as complications. Hence the choice of technique should be done considering present clinical factors as preferred by anesthesiologist.

\section{References}

1. Stamer UM, Wiese R, Stuber F, et al. Change in anaesthetic practice for Caesarean section in Germany. Acta Anaesthesiol Scand. 2005; 49:170-176. 


\section{Das of Seth: Spinal Anesthesia with General Anesthesia}

0

2. Stamer U, Schneck H, Grond S, Wulf H. Surveys on the use of regional anaesthesia in obstetrics. Curr Opin Anaesthiol. 1999;12:565571.

3. Ng K, Parsons J, Cyna AM, Middleton P. Spinal versus epidural anaesthesia for caesarean section. Cochrane Database Syst Rev 2004: 003765.

4. Practice guidelines for obstetrical anesthesia: a report by the American Society of Anesthesiologists Task Force on Obstetrical Anesthesia. Anesthesiology. 1999;90:600-611.

5. Riley ET, Cohen SE, Macario A, et al. Spinal versus epidural anesthesia for cesarean section: a comparison of time efficiency, costs, charges, and complications. Anesth Analg. 1995;80:709-712.
6. Solangi SA, Siddiqui SM, Khaskheli MS, Siddiqui MA. Comparison of the effects of general vs spinal anesthesia on neonatal outcome. Anaesth Pain Intens Care. 2012; 16:18-23.

7. Afolabi BB, Lesi FE. Regional versus general anaesthesia for caesarean section. Cochrane Database Syst Rev. 2012; 10: 004350.

8. Ajay Batra. Preference of anaesthesia for caesarean sections; spinal or general??. International Journal of Contemporary Medical Research 2016;3(10):2835-2837.

9. Veneziani A, De Tommaso O. Spinal or epidural anaesthesia for caesarean section? Compared opinions. Minerva Anestesiol. 2001;67(9 Suppl 1):169-74.

Copyright: (C) the author(s), publisher. Academia Anesthesiologica International is an Official Publication of "Society for Health Care \& Research Development". It is an open-access article distributed under the terms of the Creative Commons Attribution Non-Commercial License, which permits unrestricted non-commercial use, distribution, and reproduction in any medium, provided the original work is properly cited.

How to cite this article: Das RK, Seth M. A Comparison Study of Spinal Anesthesia with General Anesthesia in Cesarean Section. Acad. Anesthesiol. Int. 2019;4(2):30-32.

DOI: dx.doi.org/10.21276/aan.2019.4.2.8

Source of Support: Nil, Conflict of Interest: None declared. 